\title{
Evaluation of serodiagnostic tests for T.b. gambiense human African trypanosomiasis in southern Sudan
}

I.E. Elrayah, ${ }^{1}$ M.A. Rhaman, ${ }^{1}$ L.T. Karamalla, ${ }^{1}$ K.M. Khalil ${ }^{1}$ and P. Büscher ${ }^{2}$

$$
\begin{aligned}
& \text { تقيسم اختبارات التشخيص المصلي لمرض النوم البشري الإفريقي بالمثقبيات البروسية الغامبية في جنوب } \\
& \text { السودان } \\
& \text { انتصار الخاج الريح، مبارك مصطفى عبد الرحمن، لبنى تاج السر كرم الله، خليل محمد خحليل، فيليب بوشر }
\end{aligned}
$$

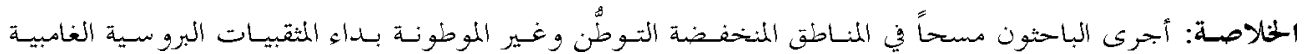

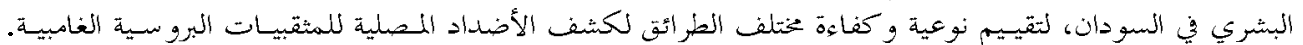

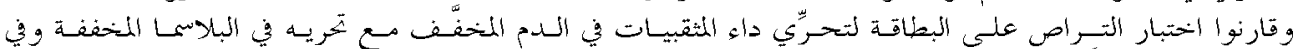

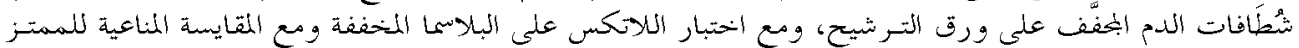

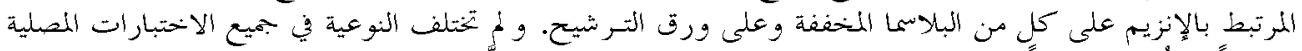

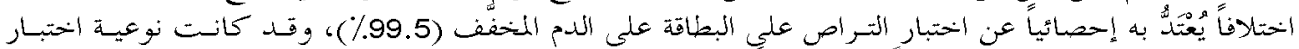

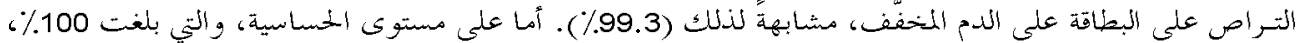

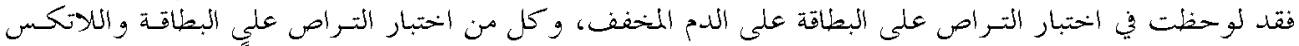

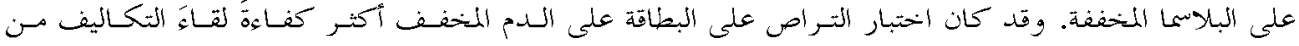

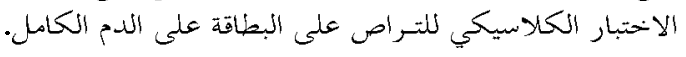

ABSTRACT A survey was conducted in a low-endemic and in a non-endemic area of Sudan to evaluate the specificity and efficiency of different serological antibody detection techniques for Trypanosoma brucei gambiense. Comparisons were made of the card agglutination test for trypanosomiasis (CATT) on diluted blood, on diluted plasma and on eluates from blood dried on filter paper, the LATEX test on diluted plasma and an ELISA on diluted plasma and filter paper. The specificities of all the serological tests were not significantly different from CATT on diluted blood $(99.5 \%)$. The specificity of CATT on diluted blood was similar (99.3\%). The highest sensitivities (100\%) were observed with CATT on diluted blood and with CATT and LATEX on diluted plasma. CATT on diluted blood was more cost-efficient than the classic test, CATT on whole blood.

\begin{abstract}
Évaluation des tests de diagnostic sérologique de la trypanosomiase humaine africaine à T.b. gambiense au Sud-Soudan

RÉSUMÉ Dans deux régions du Soudan, l'une à faible endémicité et l'autre non endémique, une enquête a été menée afin d'évaluer la spécificité et l'efficacité réelle de différentes techniques sérologiques de détection d'anticorps anti-Trypanosoma brucei gambiense. Ont été comparés le test d'agglutination sur carte pour la trypanosomiase, ou CATT (pour card agglutination test for trypanosomiasis), sur sang dilué, sur plasma dilué et sur éluats de sang séché sur papier filtre, le test d'agglutination au latex sur plasma dilué et un test ELISA sur plasma dilué et papier filtre. La spécificité de tous les tests sérologiques n'est pas apparue significativement différente de celle du CATT sur sang dilué (99,5\%), la spécificité de ce dernier étant comparable $(99,3 \%)$. Les sensibilités maximales (100\%) ont été associées au CATT sur sang dilué, ainsi qu'au CATT et au test latex sur plasma dilué. Le CATT sur sang dilué s'avère économiquement plus rentable que le test classique, à savoir le CATT sur sang total.
\end{abstract}

${ }^{1}$ Trypanosomiasis Unit, Tropical Medicine Research Institute, Khartoum, Sudan (Correspondence to I.E. Elrayah: intisar62@yahoo.com).

${ }^{2}$ Unit of Parasite Diagnostics, Department of Parasitology, Institute of Tropical Medicine, Antwerp, Belgium.

Received: 29/08/05; accepted: 31/10/05 


\section{Introduction}

Control of sleeping sickness (human African trypanosomiasis) depends on case detection by means of parasitological examination of blood, lymph or cerebrospinal fluid (CSF). Patients are given treatment only if trypanosomes have been detected in their body fluids. Mass screening of the whole population at risk for Trypanosoma brucei gambiense is currently performed using serological tests in order to select individuals carrying trypanosome-specific antibodies, on which parasitological examinations are then carried out. However, in the case of T.b. gambiense infection, parasite detection may be difficult, because of often low parasitaemia. Concentration techniques are often necessary to detect the parasites. The most commonly used serological test in the field is the card agglutination test for trypanosomiasis (CATT)/T.b. gambiense [1]. More recently another serological field test has been developed, the LATEX/T.b. gambiense [2].

Because of fluctuations in parasitaemia, the number of parasites in the blood may be very low at the time of sampling, which can give rise to the phenomenon of serologically positive, unconfirmed individuals. On the other hand, the specificity of CATT, particularly when tested on whole blood, is limited and may yield false positives. To increase the specificity of serological tests and the sensitivity of parasitological tests in the field, CATT on blood or plasma dilutions and concentration techniques - such as the haematocrit centrifugation technique (HCT) [3] or the miniature anion exchange centrifugation technique (mAECT) [4]have been proposed [5]. The use of these concentration techniques can increase the sensitivity of trypanosome detection by several orders of magnitude [6]. Recently, the applicability of CATT on blood dried on filter paper has been proposed for remote testing in the laboratory [7]. The high sensitivity and specificity of CATT on filter paper allows a better estimation of seroprevalence and incidence of the disease.

In Sudan, a surveillance of human African trypanosomiasis carried out in Eastern Equatoria and Bahr El-Jabel states during September 1998 and January-February 1999 showed a seroprevalence of $19.5 \%$ and $30.3 \%$, respectively, using CATT on whole blood [8]. A later surveillance in Bahr El-Jabel state showed an apparent seroprevalence of $43.9 \%$ [9]. In November 2001 another survey showed that the seroprevalence rate of sleeping sickness in the region was $11.1 \%$. None of these surveys detected parasites in blood smears of the CATT-seropositive persons. In a recent survey conducted in Bahr El-Jabel state in January 2003, 94 (3.96\%) of 2374 persons screened were positive using CATT on whole blood but only $9(0.38 \%)$ were positive in CATT on blood diluted by $1 / 8$, of which 3 could be confirmed parasitologically by fluid sampling and examination for lymph node pathology (LNP) and by HCT. Thus, the observed prevalence of the disease was $0.27 \%$.

According to the results obtained during the subsequent surveillance in Bahr El-Jabel state, Southern Sudan [10] it is suggested that this area is a low-endemic region of human African trypanosomiasis. Surveillance of the disease in this area should make use of a highly specific serological screening test combined with a highly sensitive parasite detection test carried out on the seropositive persons. The overall objective of this study was to evaluate the specificity and efficiency of a combination of different serological antibody detection techniques in Bahr El-Jabel state, southern Sudan. 


\section{Methods}

\section{Study areas}

A prospective cross-sectional survey was conducted in a low-endemic region for human African trypanosomiasis (Bahr El-Jabel state) and in a non-endemic area (Khartoum state).

Bahr El-Jabel state lies between longitude $30^{\circ} 30^{\prime}$ to $31^{\circ} 45^{\prime} \mathrm{E}$ and latitude $4^{\circ} 40^{\prime}$ to $5^{\circ} \mathrm{N}$. Two main types of vegetation predominate. Open savannah woodland forest and riverine gallery forest, which is interrupted by villages and numerous small plots for subsistence farming. In most cases these farms contain hedges that were deemed suitable for tsetse flies, the vector for sleeping sickness. The climate is hot throughout the year with the rains falling between March and November. Bahr ElJabel river is the most dominant feature in the area. The population at risk were visited in villages around Juba and the displacement camps in Juba town. For testing of the specificity of the antibody detection tests, Khartoum state was selected as a nonendemic area according to its situation out of the tsetse fly belt. It lies between latitude $16^{\circ} \mathrm{N}$ and $14^{\circ} \mathrm{N}$.

\section{Diagnostic techniques \\ Serological techniques}

- CATT/T.b. gambiense: CATT is an antibody detection test based on direct agglutination of specific antibodies with antigens exposed at the surface of fixed, stained trypanosomes of variable antigen type T.b. gambiense LiTat 1.3. The test was performed as described by the manufacturer [1].

- CATT/diluted blood (CATT/DB): CATT performed on 2-fold serial dilutions of blood starting from $1 / 4$ up to $1 / 32$. The test was also performed on plasma (CATT/PL) [5].
- CATT/filter paper (CATT/FP): CATT performed on blood eluted from filter paper [7].

- LATEX/T.b. gambiense is an antibody detection test based on indirect agglutination of specific antibodies with antigens exposed at the surface of latex beads [2]. The test was performed according to the manufacturer's instructions. The reagent consisted of a mixture of variable surface glycoproteins of T.b. gambiense variable antigen type 1.3, 1.5 and 1.6 coupled to latex particles. In this study, the test was used on 2-fold serial dilutions of plasma.

- ELISA/T.b. gambiense [11] is an antibody detection test based on reaction of specific antibodies with the same antigens as in LATEX, but fixed in an ELISA plate. In this study, the test was used on diluted plasma (ELISA/PL) with the cut-off optical density set at 0.771 and on blood eluted from filter paper (ELISA/FP) with the cut-off optical density set at 0.416 .

Parasitological techniques

- LNP examination: lymph aspirates were obtained from enlarged cervical lymph nodes and examined by light microscope for the presence of the parasite [12].

- HCT: heparinized capillary tubes (4 capillaries of $60 \mu \mathrm{L}$ ) were filled with venous blood, sealed at one end with Plasticine putty and spun for 6-8 min in a microhaematocrit centrifuge (12000 rpm) and examined at $10 \times 10$ magnification under the microscope [3].

- mAECT: a kit designed for use in the field. A sample of $300 \mu \mathrm{L}$ of heparinized blood can be processed in 1 column. This test was considered to be the most sensitive for trypanosome detection in the field [4]. The test was performed 
according to the manufacturer's instructions.

- CSF examination: samples were obtained by lumbar puncture $(5 \mathrm{~mL})$. About $1 \mu \mathrm{L}$ of CSF was immediately processed for cell counts in a Kova counting chamber. The rest was transferred in a sealed glass Pasteur pipette and centrifuged at low speed $(3000 \mathrm{~g})$ for $10 \mathrm{~min}$. After centrifugation, the Pasteur pipette was mounted in a special viewing chamber for direct examination of the sediment under the microscope $(10 \times 10$ magnifications) for the presence of trypanosomes.

\section{Sample}

Every person resident in the selected study area was in principle eligible. The study included persons of any age and sex for whom informed consent to participate in the study was obtained. The exclusion criteria were a previous history of human African trypanosomiasis or unwilling to participate.

\section{Endemic area}

In the endemic area, active surveillance was conducted in April and October 2003 in 2 camps for displaced people in Juba (Sindru and Bungu camps) and in 3 villages west of Juba (Koda, Luri, Kabo). For ethical reasons, all persons in the displaced camps and in the villages willing to participate were included in the study, without considering pre-fixed sample sizes: 1381 persons participated after signing the informed consent form (they included 31 people identified as CATT whole-blood seropositive in previous surveillances in Bahr El-Jabel state). A further 58 persons presenting themselves at Juba hospital with signs and/or symptoms suggestive of human African trypanosomiasis were included in the study as passive cases.

\section{Non-endemic area}

To test the specificity of the antibody detection tests, plasma samples were collected in the non-endemic area from 203 volunteers and blood donors at the blood transfusion bank.

\section{Data collection}

Prior to the study, standard operating procedures and case report forms were prepared.

After participants had signed the informed consent form, all the study subjects were clinically examined for presence of enlarged cervical lymph nodes by the medical doctor. From all suspected cases with enlarged lymph nodes, the lymph node aspirate was microscopically examined for presence of trypanosomes.

From all participants, blood was taken by fingerprick in 2 heparinized capillary tubes $(2 \times 60 \mu \mathrm{L}$ of blood $)$. From 1 capillary tube, the blood was diluted (1/4) and tested by CATT/DB to assess the end-titre in CATT. From the other capillary tube, the blood was collected on filter paper for testing the CATT/FP and ELISA/FP. A sample of venous blood on heparin was also taken for the preparation of plasma.

From all suspected cases with CATT/ DB end-titre $\geq 1: 4$ (seropositive), a sample of $5 \mathrm{~mL}$ of venous blood on heparin was taken. The blood was examined immediately for presence of trypanosomes by HCT and mAECT. The rest of the blood was centrifuged for preparation of plasma which was frozen for further serological testing in the laboratory in Khartoum (CATT/PL, LATEX/PL, ELISA/PL).

On the parasitologically confirmed patients, a lumbar puncture was done and 5 $\mathrm{mL}$ of CSF collected to determine the stage. The CSF was examined immediately for cell count and for presence of trypanosomes in the sediment after centrifugation. The su- 
pernatant of centrifuged CSF was frozen for further analyses in the laboratory. Patients with $>5$ cells $/ \mu \mathrm{L}$ or trypanosomes in the CSF were considered to be in the second stage.

Demographic, clinical, serological and parasitological data and disease stage were entered in the case report forms as well as the code of the biological samples collected from the subjects. Data collections were supervised by the medical doctor in the field and by the principal investigator. Case report forms were collected by the principal investigator for further completion with data obtained in the serological laboratory tests. Case report forms were double entered in an electronic database for further data analysis.

\section{Data management and statistical analysis}

The specificity of the serological tests was calculated with the confirmed human African trypanosomiasis cases as true positives and the healthy non-endemic controls as true negatives. Specificities of the serological tests were compared by the McNemar $\times 2$ test.
Estimation of the cost-efficiency of each test or test combinations was calculated and compared in order to propose an improved diagnostic decision tree for human African trypanosomiasis surveillance in Southern Sudan. The costs were calculated including only consumables and reagents; costs related to equipment, small laboratory instruments and fieldwork were excluded. For comparison with earlier studies, the cost of CATT performed on whole blood was also calculated.

SPSS was used for statistical analysis.

\section{Results}

\section{Non-endemic area}

The serological results from the 203 plasma samples collected in the non-endemic area are presented in Table 1. All participants showed negative reactions with CATT on filer paper (CATT/FP). The specificity of this test was therefore $100 \%$. On diluted blood at $1 / 4$ dilution (CATT/DB) and on plasma (CATT/PL) the tests were $99.5 \%$ specific. LATEX/PL was $99.0 \%$ specific at plasma dilution $1 / 8$. The specificity of both

\begin{tabular}{|c|c|c|c|c|c|}
\hline$\overline{\text { Test }}$ & $\begin{array}{l}\text { Dilution } \\
\text { or cut-off }\end{array}$ & $\begin{array}{c}\text { Total no. } \\
\text { of samples } \\
\text { tested }\end{array}$ & $\begin{array}{c}\text { No. of } \\
\text { positives }\end{array}$ & $\begin{array}{c}\text { Specificity } \\
(\%)\end{array}$ & $95 \% \mathrm{Cl}$ \\
\hline CATT/DB & $1 / 4$ & 203 & 1 & 99.5 & $97.3-100.0$ \\
\hline CATT/FP & $\mathrm{n} / \mathrm{a}$ & 203 & 0 & 100.0 & $98.5-100.0$ \\
\hline CATT/PL & $1 / 8$ & 203 & 1 & 99.5 & $97.3-100.0$ \\
\hline LATEX/PL & $1 / 8$ & 203 & 2 & 99.0 & $96.5-99.9$ \\
\hline ELISA/PL & 0.771 & 200 & 3 & 98.5 & $95.7-99.7$ \\
\hline ELISA/FP & 0.416 & 196 & 3 & 98.5 & $95.6-99.7$ \\
\hline
\end{tabular}

CATT = card agglutination test for trypanosomiasis; ELISA = enzyme-linked immunosorbent assay; $D B=$ diluted blood; $F P=$ filter paper; $P L=$ plasma $\mathrm{Cl}=$ confidence interval; $\mathrm{n} / \mathrm{a}=$ not applicable. 
ELISA on plasma samples (ELISA/PL) and on filter paper eluates (ELISA/FP) was $98.5 \%$.

When comparing CATT/DB with all the other serological tests by a $2 \times 2$ table $\left(\chi^{2}\right.$ with continuity correction), none of the tests was significantly different from CATT/DB $(P>005)$

\section{Endemic area}

Active surveillance

Out of the 1381 people examined in the endemic area, 13 were seropositive in CATT/ DB thus showing a seroprevalence of $0.94 \%$ : 3 out of them were parasitologically confirmed, 2 in lymph node puncture and 1 in HCT, showing a prevalence of $0.20 \%$. All 3 parasitologically confirmed patients were seropositive in LATEX/PL and CATT/PL, 2 were negative in CATT/FP and 1 was negative in both ELISA tests. Considering the 10 non-parasitologically confirmed seropositive samples as false positives, the specificity of CATT/DB at 1/4 dilution carried out in the field was $99.3 \%$ [95\% confidence interval (CI): 98.7-99.7] which is very similar to the specificity observed in the non-endemic region (Table 2).
Although there was a selection bias in the sampling towards CATT/DB positivity, the specificity of CATT/FP can be calculated as $99.7 \%$ (95\% CI: 99.1-99.7) in the low-endemic area Bahr El-Jabel state. For the other tests, the number of samples was too small compared to the selection bias for calculating the specificity.

When comparing CATT/DB with all the other serological tests by a $2 \times 2$ table $\left(\chi^{2}\right.$ with continuity correction), only CATT/PL was different from CATT/DB $(P=0.004)$.

\section{Passive surveillance}

A total of 58 persons presented themselves to Juba teaching hospital as suspected cases: 3 cases were found parasitologically positive, 2 of them diagnosed positive by detecting trypanosomes in their lymph node aspirate, 1 diagnosed by detecting trypanosomes in the cerebrospinal fluid. A further 3 seropositive cases were considered patients for treatment due to their clinical signs and high numbers of cells in their CSF. All patients were found in the second stage of the disease $(>5$ cells $/ \mu \mathrm{L}$ or trypanosomes in the CSF). They received the appropriate treatment.

\begin{tabular}{|c|c|c|c|c|c|c|c|}
\hline$\overline{\text { Test }}$ & $\begin{array}{l}\text { Dilution } \\
\text { or cut-off }\end{array}$ & $\begin{array}{c}\text { Total } \\
\text { no. of } \\
\text { samples } \\
\text { tested }\end{array}$ & $\begin{array}{l}\text { No. of positi } \\
\text { Parasitologically } \\
\text { confirmed, } \\
\text { CATT/DB } \\
\text { positive }\end{array}$ & $\begin{array}{l}\text { ive results/no. } \\
\text { Seropositives } \\
\text { in CATT/DB, } \\
\text { non-patient }\end{array}$ & $\begin{array}{l}\text { of samples } \\
\text { Seronegatives } \\
\text { in CATT/DB, } \\
\text { non-patient }\end{array}$ & $\begin{array}{c}\text { Specificity } \\
(\%)\end{array}$ & $\begin{array}{c}P \text { - } \\
\text { value }\end{array}$ \\
\hline CATT/FP & $\mathrm{n} / \mathrm{a}$ & 772 & $1 / 3$ & $1 / 5$ & $1 / 764$ & 99.4 & 0.130 \\
\hline CATT/PL & $1 / 8$ & 61 & $3 / 3$ & $6 / 7$ & $12 / 51$ & - & 0.004 \\
\hline LATEX/PL & $1 / 8$ & 53 & $5 / 3$ & $1 / 3$ & $4 / 47$ & - & 0.675 \\
\hline ELISA/PL & 0.771 & 61 & $2 / 3$ & $2 / 6$ & $7 / 52$ & - & 0.761 \\
\hline ELISA/FP & 0.416 & 58 & $2 / 3$ & $2 / 5$ & $7 / 50$ & - & 0.527 \\
\hline
\end{tabular}

- = not available.

CATT = card agglutination test for trypanosomiasis; $E L I S A=$ enzyme-linked immunosorbent assay; $D B=$ diluted blood; $F P=$ filter paper; $P L=$ plasma.

n/a= not applicable. 
Only 2 of the 6 patients were positive by CATT/DB, indicating a low sensitivity of CATT/DB in this setting. On the other hand, all the 5 patients tested with CATT/ PL were positive. This may implicate that the specificity of CATT/PL is higher than calculated during active surveillance and that the specificity and sensitivity of the other tests, particularly CATT/DB and CATT/FP, are lower than observed during active surveillance.

\section{Cost-efficiency of diagnostic strategy}

Looking at the costs of the serological tests, we observed that the difference between CATT on whole blood (the classic test) and CATT on diluted blood was minimal (US\$ 0.50 and 0.53 per person). Collecting samples on filter paper or as plasma does increase the cost considerably for the agglutination tests (up to US\$ 0.80 per person for CATT/FP, CATT/PL and LATEX/PL). When plasma or filter paper eluates were tested by ELISA, costs were even lower for ELISA/FP (US\$ 0.47) than for CATT on whole blood (Table 3).

Concerning the parasitological tests, LNP and HCT were cheap (US\$ 0.21 and 0.09 ) compared with the mAECT (US\$ 3.11). However, LNP can only be performed on patients presenting with swollen cervical lymph nodes. Lumbar puncture, seldom performed for parasitological confirmation but always needed for stage determination, costs US\$ 1.34 , mainly consisting of the price of a disposable lumbar puncture needle.

Taking into account the costs of the serological screening tests and the parasitological confirmation tests, we calculated the costs to detect and stage a patient starting with CATT/whole blood and CATT/DB as screening test. For this calculation, we used

\begin{tabular}{lc}
\hline $\begin{array}{l}\text { Table } 3 \text { Costs per person of serological } \\
\text { and diagnostic tests for human African } \\
\text { trypanosomiasis including only reagents and } \\
\text { consumables }\end{array}$ \\
\hline Test & Cost per person (US\$) \\
\hline Serological tests & \\
CATT/whole blood & 0.50 \\
CATT/DB & 0.53 \\
CATT/FP & 0.79 \\
CATT/PL & 0.80 \\
LATEX/PL & 0.80 \\
ELISA/PL & 0.62 \\
ELISA/FP & 0.47 \\
Parasitological tests & \\
LNP & 0.21 \\
HCT & 0.09 \\
mAECT & 3.11 \\
Lumbar puncture & 1.34 \\
\hline
\end{tabular}

CATT = card agglutination test for trypanosomiasis; ELISA = enzyme-linked immunosorbent assay; $D B=$ diluted blood; $F P=$ filter paper; $P L=$ plasma.

$L N P=$ lymph node fluid sampling and examination;

$H C T=$ haematocrit centrifugation technique; $m A E C T=$ mini-anion exchange centrifugation technique.

the data obtained in the active surveillance study (1381 persons screened, 3 patients confirmed) with the following assumptions:

- The sensitivity of both CATT/whole blood and CATT/DB is $100 \%$.

- The specificity of CATT/whole blood is $96.3 \%$ (based on the seroprevalence of $3.76 \%$ with no confirmed patients in the survey of January 2003).

- All seropositive persons have to undergo all parasitological tests for confirmation diagnosis.

From Table 4, we observe that the difference in costs between the 2 active surveillance strategies was about US\$ 50 per detected patient within a population of 1381 in the low-endemic Baher El-Jabel state. 
Table 4 Costs to find 1 patient with human African trypanosomiasis in an endemic area by active surveillance, comparing the card agglutination test for trypanosomiasis (CATT) on whole blood and diluted blood (DB)

\begin{tabular}{|c|c|c|}
\hline Variable & $\begin{array}{l}\text { CATT/ } \\
\text { whole } \\
\text { blood }^{\mathrm{a}}\end{array}$ & $\mathrm{CATT} / \mathrm{DB}^{\mathrm{b}}$ \\
\hline Screened population (No.) & 1381 & 1381 \\
\hline Seropositive cases (No.) & 54 & 13 \\
\hline True positive cases (No.) & 3 & 3 \\
\hline False positive cases (No.) & 51 & 10 \\
\hline True negative cases (No.) & 1327 & 1368 \\
\hline False negative cases (No.) & 0 & 0 \\
\hline Specificity of test (\%) & 96.3 & 99.3 \\
\hline Sensitivity of test (\%) & 100.0 & 100.0 \\
\hline \multicolumn{3}{|l|}{ Prices of tests (US\$) } \\
\hline CATT (on population) & 686.4 & 734.2 \\
\hline LNP (on seropositives) & 11.3 & 2.7 \\
\hline $\begin{array}{l}\text { HCT (on seropositives) } \\
\text { mAECT (on }\end{array}$ & 4.7 & 1.2 \\
\hline seropositives) & 166.6 & 40.4 \\
\hline $\begin{array}{l}\text { Lumbar puncture (on } \\
\text { seropositives) }\end{array}$ & 72.0 & 17.5 \\
\hline Total prices of tests & 941.0 & 796.0 \\
\hline $\begin{array}{l}\text { Price to find and stage } \\
1 \text { patient }\end{array}$ & 313.7 & 265.3 \\
\hline \multicolumn{3}{|c|}{$\begin{array}{l}{ }^{a} \text { Real data; }{ }^{b} \text { Hypothetical data based on } 96.3 \% \\
\text { specificity. } \\
\text { HCT = haematocrit centrifugation technique; } L N P= \\
\text { lymph node fluid sampling and examination; } m A E C T= \\
\text { mini-anion exchange centrifugation technique. }\end{array}$} \\
\hline
\end{tabular}

\section{Discussion}

The data collected in the non-endemic region show that all serological tests used in this study have similar specificity. This implies that the choice between the different tests for use in an endemic region will depend on other parameters such as sensitivity, cost and applicability in the field. Regarding the latter, only CATT/DB, CATT/PL and LATEX/PL are suitable. All the other tests are laboratory-based and may find their use for monitoring seroprevalence in endemic regions.

From the active and passive surveillance study in the endemic region, we conclude that there is no significant difference in the specificity of CATT/DB and CATT/FP but that the sensitivity becomes important when choosing the most appropriate screening test. Indeed, CATT/FP is less sensitive than CATT/DB, which in turn is less sensitive than CATT/PL. The low sensitivity of CATT/FP is unexpected, when compared to the results obtained by Chappuis et al. with this test [7]. It cannot be excluded that this low sensitivity of CATT/FP is not intrinsic but related to the logistic conditions during the passive surveillance study.

When we compared the seroprevalence rate $(3.76 \%)$ of the previous surveillance using CATT on whole blood [10] with the seroprevalence rate $(0.94 \%)$ in this study using CATT on diluted blood, we observed that the number of seropositive cases was greatly reduced and decreased the workload on the parasitological testing to confirm cases.

The other serological tests used in the laboratory-LATEX/PL, ELISA/PL and ELISA/FP — showed high specificity in the non-endemic area of Khartoum. Nevertheless, they showed slightly lower specificity in Bahr El-Jabel state (low-endemic area), perhaps due to the small number of samples tested in the laboratory. Further studies should focus on the sensitivity of these tests. These studies may include more extensive testing of ELISA to evaluate its value for monitoring seroprevalence [11].

When the costs to find 1 patient in a lowendemic region are taken into account, it appears that CATT/DB is more cost-efficient than the classic test, CATT/whole blood, with a difference of US\$ 50 per patient. We can thus propose replacing CATT/ 
whole blood by CATT/DB for screening of the population in low-endemic regions, even though the CATT/DB is slightly more complicated to perform in the field (using diluted blood).

Taking into account the observed low sensitivity of CATT/DB in the passive surveillance study, we can propose the CATT/PL for screening the population at risk. Indeed, if we accept that the sensitivity and specificity of CATT/PL are $100 \%$ and 99.5\% respectively, the CATT/PL would be extremely cost-efficient. If plasma is prepared from blood taken in a capillary from the finger and sedimented in a microtitre plate, CATT/PL is no more complicated than CATT/DB to perform in the field.
For the time being, we can recommend using CATT/DB instead of CATT/whole blood as the screening test of choice in lowendemic T.b. gambiense sleeping sickness regions.

\section{Acknowledgement}

This investigation received technical and financial support from the joint WHO Eastern Mediterranean Region (EMRO), Division of Communicable Diseases (DCD) and the WHO Special Programme for Research and Training in Tropical Diseases (TDR): the EMRO/TDR Small Grants Scheme for Operational Research in Tropical and Other Communicable Diseases.

\section{References}

1. Magnus E, Vervoort T, Van Meirvenne N. A card agglutination test with stained trypanosomes (CATT) for the serological diagnosis of T.b. gambiense. Annales de la Société belge de Médecine Tropicale, 1978, 58:169-76.

2. Büscher $P$ et al. Improved latex agglutination test for detection of antibodies in serum and cerebrospinal fluid of Trypanosoma brucei gambiense infected patients. Acta tropica, 1999, 73:11-20.

3. Baker JR. Techniques for the detection of trypanosome infections. In: Mulligan HW, ed. The African trypanosomiases. London, George Allen Unwin, 1970:67.

4. Lumsden $\mathrm{WH}$ et al. Trypanosoma brucei: miniature anion-exchange centrifugation technique for detection of low parasitaemias: adaptation for field use. Transactions of the Royal Society of Tropical Medicine and Hygiene, 1979, 73:312-7.

5. Simarro PP et al. Attitude towards CATTpositive individuals without parasitological confirmation in the African trypanosomiasis (T.b. gambiense) focus of Quiçama
(Angola). Tropical medicine and international health, 1999, 4:858-61.

6. Control and surveillance of African trypanosomiasis. Report of a WHO Expert Committee. Geneva, World Health Organization, 1998 (WHO Technical Report Series, No. 881).

7. Chappuis F et al. Field evaluation of the CATT/Trypanosoma brucie gambiense on blood-impregnated filter papers for diagnosis of human African trypanosomiasis in southern Sudan. Tropical medicine and international health, 2002, 7(11):942-8.

8. El Rayah IE et al. Prevalence of sleeping sickness in Sudan. In: Proceedings of the 25th International Scientific Council for Trypanosomiasis Research and Control (ISCTRC), Mombasa, Kenya, 27 Sept-1 Oct 1999:107-10 (Publication No. 121).

9. EI Rayah IE. Technical report on Sleeping sickness and vector control in Equatoria region (Eastern Africa Network for Trypanosomosis Report). In: Proceedings of the 26th International Scientific Council for Trypanosomiasis Research and Con- 
trol (ISCTRC), Ouagadougou, Burkina Faso, 1-5 Oct, 2001.

10. El Rayah IE et al. Updating the situation of sleeping sickness in Bahr El Jabel State, Southern Sudan. In: Proceedings of the 27th International Scientific Council for Trypanosomiasis Research and Control (ISCTRC), Pretoria, South Africa, 29 Sept-4 Oct, 2003 (Publication No. 122).

11. Büscher $P$ et al. A serodiagnostic ELISA using variable antigens of Trypanosoma brucei gambiense. In: Sones KR, ed. Proceedings of the 22nd Meeting of the International Scientific Council for Trypanosomiasis Research and Control (ISCTRC), Kampala, Uganda, 25-29 October 1993. Nairobi, Scientific, Technical and Research Commission of the Organization of African Unity, 1995:46-52

12. Epidemiology and control of African trypanosomiasis. Report of a WHO Expert Committee. Geneva, World Health Organization, 1997 (WHO Technical Report Series, No. 739).

\section{Human African trypanosomiasis: role of WHO}

The resurgence of sleeping sickness since the 1970s led WHO to reinforce its human African trypanosomiasis programme. The WHO programme provides support and technical assistance to national control programmes. A network has been established including donor countries, private foundations, MGOs, regional institutions, research centres and universities to participate in surveillance and control, and to undertake research projects for the development of new drugs and diagnostic tools.

The objectives of the WHO Programme are to: strengthen and coordinate control measures and ensure field activities are sustained; strengthen existing surveillance systems; support monitoring of treatment and drug resistance through the network; develop an information database and implement training activities; promote inter-agency collaboration with the Food and Agriculture Organisation (FAO) and the International Atomic Energy Agency (IAEA). This agency is dealing with vector control through male flies made sterile by radiation. 